Short communication

\title{
Proteomic profiling reveals candidate markers for arsenic-induced skin keratosis ${ }^{\text {मr }}$
}

\author{
Zhiling Guo ${ }^{a}$, Qin Hu ${ }^{a}$, Jijing Tian a, Li Yan a, Chuanyong Jing a, Heidi Qunhui Xie a ,

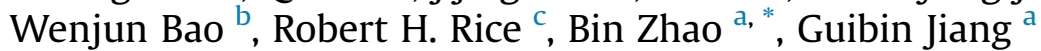 \\ ${ }^{a}$ Research Center for Eco-Environmental Sciences, Chinese Academy of Sciences, Beijing 100085, China \\ b JMP Life Sciences, SAS Institute, Cary, NC 27513, USA \\ c Department of Environmental Toxicology, University of California, Davis, CA 95616-8588, USA
}

\section{A R T I C L E I N F O}

\section{Article history:}

Received 27 April 2016

Received in revised form

20 June 2016

Accepted 5 August 2016

Available online 20 August 2016

\section{Keywords:}

Proteomic

Arsenic

Biomarker

Skin keratosis

Human epidermis

\begin{abstract}
A B S T R A C T
Proteomics technology is an attractive biomarker candidate discovery tool that can be applied to study large sets of biological molecules. To identify novel biomarkers and molecular targets in arsenic-induced skin lesions, we have determined the protein profile of arsenic-affected human epidermal stratum corneum by shotgun proteomics. Samples of palm and foot sole from healthy subjects were analyzed, demonstrating similar protein patterns in palm and sole. Samples were collected from the palms of subjects with arsenic keratosis (lesional and adjacent non-lesional samples) and arsenic-exposed subjects without lesions (normal). Samples from non-exposed healthy individuals served as controls. We found that three proteins in arsenic-exposed lesional epidermis were consistently distinguishably expressed from the unaffected epidermis. One of these proteins, the cadherin-like transmembrane glycoprotein, desmoglein 1 (DSG1) was suppressed. Down-regulation of DSG1 may lead to reduced cellcell adhesion, resulting in abnormal epidermal differentiation. The expression of keratin 6c (KRT6C) and fatty acid binding protein 5 (FABP5) were significantly increased. FABP5 is an intracellular lipid chaperone that plays an essential role in fatty acid metabolism in human skin. This raises a possibility that overexpression of FABP5 may affect the proliferation or differentiation of keratinocytes by altering lipid metabolism. KRT6C is a constituent of the cytoskeleton that maintains epidermal integrity and cohesion. Abnormal expression of KRT6C may affect its structural role in the epidermis. Our findings suggest an important approach for future studies of arsenic-mediated toxicity and skin cancer, where certain proteins may represent useful biomarkers of early diagnoses in high-risk populations and hopefully new treatment targets. Further studies are required to understand the biological role of these markers in skin pathogenesis from arsenic exposure.
\end{abstract}

() 2016 Elsevier Ltd. All rights reserved.

\section{Introduction}

Arsenic, one of the most toxic metalloids, is widely encountered in the environment arising naturally and from pharmaceutical, agricultural and industrial applications (Mead, 2005) that can appear in groundwater. Globally, millions of people are suffering from exposure to hazardous concentrations of arsenic in their water supplies (Wang et al., 2002). Arsenic-induced skin lesions are endemic in many remote mountainous regions in China, especially

\footnotetext{
This paper has been recommended for acceptance by von Hippel Frank A.

* Corresponding author. 18 Shuangqing Rd, Beijing 100085, China.

E-mail address: binzhao@rcees.ac.cn (B. Zhao).
}

in the Shanyin district (Guo et al., 2003). Average arsenic concentrations in groundwater there are nearly 17 times higher than the United States Environmental Protection Agency guideline of $10 \mu \mathrm{g} /$ $\mathrm{L}$, and typical clinical features of hyperkeratosis of palms and soles are observed (Cui et al., 2013). Previous work has found that individuals in this region had higher arsenic content in hair and nail than the average Chinese, consistent with arsenic induction of dermatological lesions (Cui et al., 2013). Those people with noncancerous skin lesions are at high risk of developing skin and internal cancers (Mead, 2005). Epidemiologic studies have revealed that chronic arsenic exposure can induce cancer and a host of adverse health effects in the digestive, respiratory, cardiovascular and nervous systems (Matschullat, 2000).

Possible mechanisms of arsenic action have been proposed, 
including arsenic-induced oxidative stress, chromosomal damage, altered transcription factor levels, and impaired DNA repair (Yu et al., 2006). However, further details of arsenic's mechanism of action still need to be elucidated. Identifying reliable molecular markers will be valuable for understanding the manifestations of arsenic-induced skin disease, a challenge that emerging omics technologies could meet (Vlaanderen et al., 2010). Proteomic analysis can help to identify proteins that are differentially expressed in afflicted tissue, which may reveal useful diagnostic markers or even drug targets. Rodent and cell models have provided valuable information on arsenic action (Tokar and Waalkes, 2011), but studies of human samples are urgently needed. Research on biomarkers of arsenic-exposed human samples, such as skin lesions, is very limited. Previous studies have analyzed arsenic-exposed human serum and urinary proteomic profiles and identified several potential biomarkers (Harezlak et al., 2008; Hegedus et al., 2008; Zhai et al., 2005). According to the IARC, skin shows the strongest association between chronic arsenic exposure and cancer (IARC, 2004), and dermal manifestations such as hyperpigmentation and hyperkeratosis are diagnostic of chronic arsenicosis. Arsenical hyperkeratosis appears predominantly on the palms of the hands and soles of the feet (Liu et al., 2002).

Two-dimensional gel electrophoresis has been applied in arsenic-related proteomic studies (Yu et al., 1993), but this method has the disadvantages of low reproducibility, poor representation of low abundant proteins, inaccurate quantification, high labor and time requirements, and inability to analyze the membrane and hydrophobic proteins (Abdallah et al., 2012). Shotgun proteomics, which utilizes reverse phase separation of peptides instead of electrophoretic protein separation, has higher speed, sensitivity, accuracy, and throughput with lower sample consumption (Zhang et al., 2013). In this study, human palm samples from arsenicexposed skin-diseased subjects (lesional and non-lesional epidermis), arsenic-exposed normal subjects (normal) and nonexposed healthy subjects (control) were obtained for shotgun proteomic analysis. The response of palm to arsenic was investigated by comparing protein expression profiles of lesional epidermis with unaffected epidermis with the aim of finding alterations associated with arsenic exposure. Protein alterations may be useful biomarkers for early diagnoses and may suggest new treatment targets. In addition, healthy foot epidermal samples were also collected to determine the degree of difference between human palm and foot skin in healthy subjects.

\section{Materials and methods}

\subsection{Study participants}

This study was approved by the Institutional Review Board at the Research Center for Eco-Environmental Sciences, Chinese Academy of Sciences. All affected and healthy subjects or their legal guardians provided written and informed consent. A total of 21 individuals (Table S1) were recruited for sampling and were all checked by a physician to ensure the presence or absence of skin disease. Arsenic-exposed diseased palm samples (lesional and adjacent non-lesional samples from the same palm) were collected from six individuals who resided in the arsenic-contaminated area in Shanyin district throughout their lives and had severe skin lesions. Arsenic-exposed unaffected palm samples (normal) were obtained from eight individuals from the same place in the Shanyin district without skin pathologic symptoms. Non-exposed samples (control) were obtained from seven individuals with no prior history of arsenic exposure or any skin disease. For the palm and foot sole comparison, additional foot samples were collected from the normal and control groups.
Stratum corneum from palms and foot soles of subjects were collected by applying a 22-mm diameter tape circle from D-Squame Pro Kits (CuDerm Corp, Dallas, TX) to the skin with circular pressure after fully cleaning the sampling sites (Rice et al., 2013). During the period of sampling, samplers always wore clean disposable gloves and masks. Samples were immediately transferred to sterile $15 \mathrm{~mL}$ plastic tubes to minimize any contamination from air. They were incubated in a solution of $2 \%$ sodium dodecyl sulfate- $0.1 \mathrm{M}$ sodium phosphate (SDS- $\mathrm{NaH}_{2} \mathrm{PO}_{4}, \mathrm{pH} 7.8$ ) at room temperature for 2 days to detach the cells. Cell pellets were harvested, and re-suspended in $0.4 \mathrm{~mL}$ of SDS-phosphate buffer. Protein disulfides were reduced in $25 \mathrm{mM}$ dithioerythritol and alkylated with $50 \mathrm{mM}$ iodoacetamide. Proteins were precipitated with $1 \mathrm{~mL}$ of $100 \%$ ethanol, rinsed twice with $70 \%$ ethanol, and then lysed in fresh $0.1 \mathrm{M}$ ammonium bicarbonate containing $10 \%$ acetonitrile and $1 \%$ (by weight) TPCKtreated reductively methylated trypsin at room temperature for 3 days. Complete digestion was verified by centrifugation of digests at $1000 \mathrm{rpm}$ for $3 \mathrm{~min}$, with no pellet observed. Samples obtained were adjusted to approximately equal peptide concentration by A280, acidified with $0.1 \%$ trifluoroacetic acid, and then electrosprayed into a liquid chromatography tandem mass spectrometer (LC-MS/MS) for further fractionation and identification.

\subsection{Protein identification}

Sample preparation was carried out as previously reported (Rice et al., 2013). Samples were analyzed by LC-MS/MS using a ThermoFinnigan LTQ ion trap mass spectrometer coupled with electrospray ionization (ESI) source interfaced with a C18 reverse-phase chromatography column. Samples were run in a block randomized order. Details of sample processing and protein identification were as previously described (Hu et al., 2013).

\subsection{Statistical analysis}

Spectral counting data were analyzed using JMP Genomics 8.0 software (http://www.jmp.com/en_us/software/jmp-genomics. html, SAS Institute Inc., Cary, NC). The data were imported into JMP Genomics and were log2 transformed with shifting factor as 1 . Shifting factor was added back into the data after standardization, avoiding missing values resulting from the data being set to 0 after standardizing data in further log transformation. The log transformed data were normalized by quantile normalization, which normalizes data by aligning ranked columns, computing their mean, and then replacing the original data with the average quantiles. It guarantees identical marginal univariate densities and distribution of each sample (Bolstad et al., 2003). After quantile normalization, significantly differentially expressed proteins for different conditions were identified by ANOVA modeling. The ANOVA model included the subject age, samples from left/right hand, physical condition, exposure years, and sex as fixed effects, and block as random effect. The criteria for selection of significant differential expressed proteins were a fold change $>1.5$ and a $p$ value $<0.05$.

\subsection{Bioinformatic analysis}

Protein ontology classification was performed by importing the proteins into the protein analysis through evolutionary relationships (PANTHER) classification system (http://www.pantherdb.org/ , version 10.0). The proteins were grouped according to their molecular functions, biological processes and protein classes. 


\section{Results and discussion}

\subsection{Similar protein patterns between hand and foot}

Shotgun proteomic analysis of samples from both hands and feet from 15 individual subjects ( 7 for control and 8 for normal subjects) permitted identification of 74 proteins (Table S2) by the stringent criteria employed. The proteins were divided into categories based on protein classes, molecular functions, and biological processes using the PANTHER classification system (Fig. 1A-C). Protein class analysis indicated the major portion of the identified proteins belonged to cytoskeletal (37.5\%) and structural categories (32.5\%). Keratins comprised more than one-quarter of the proteins. Among the keratins identified, the most prominent were KRT1, KRT9, KRT10, KRT2, KRT14, KRT16, KRT6C; prominent among the non-keratins were junctional proteins (DSG1, DSP, JUP, DSC1, PKP1), transglutaminase substrates (KPRP, FLG) and related proteins (FLG2, HRNR), consistent with a previous report and with their location in the outermost cornified layer (Rice et al., 2013). The dominant molecular functions that the identified proteins were involved in were structural molecule (59\%), followed by catalytic

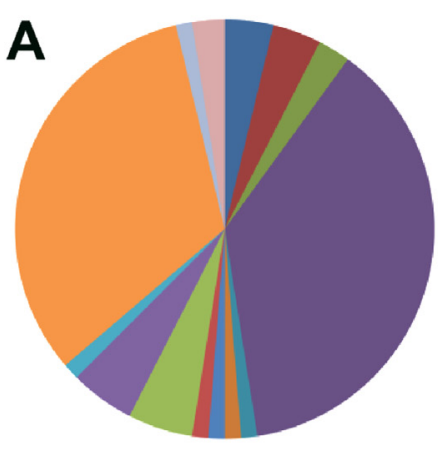

alcium-binding protein - cell adhesion molecule - cell junction protein - cytoskeletal protein nenzyme modulator nydrolase - ligase - lyase oxidoreductase n signaling molecule a storage protein structural protein w transfer/carrier protein ntransferase

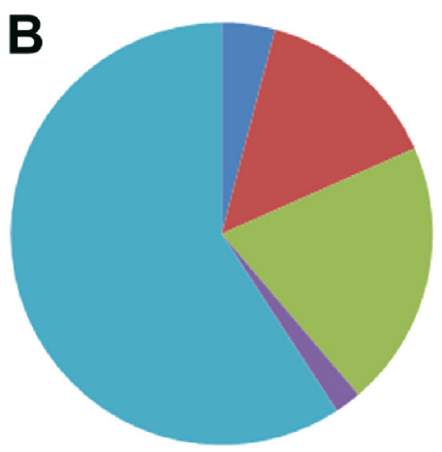

antioxidant activity a binding catalytic activity n enzyme regulator activity w structural molecule activity

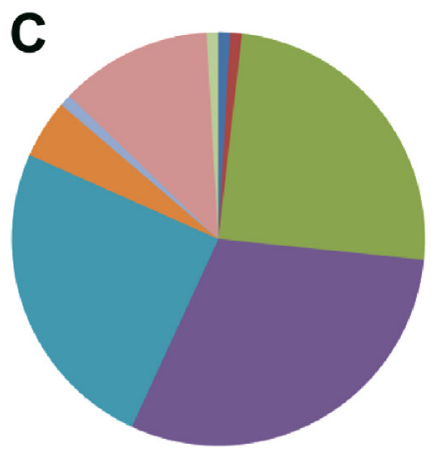

a biological adhesion - biolgical regulation - cellular component organization - cellular process

a developmental process immune system process - localization

metabolic process response to stimulus

Fig. 1. Functional classifications of the identified proteins according to their (A) protein classes, (B) molecular functions and (C) biological processes using PANTHER classification system. activity (20.0\%) and binding activity (14.0\%). The biological processes included cellular process (30.3\%), cellular component organization (24.8\%), developmental process (24.8\%), and metabolic process (11.9\%).

In both control and normal groups, the most prevalent proteins in palm showed little differences in spectral counts from foot sole. Exceptions were structural proteins such as KRT16, KRT17, and KRT6C where expression was higher in foot sole in both groups (Fig. 2, Table S3), perhaps reflecting a protective role of these structural molecules.

Skin is a major barrier protecting the body from physical, chemical and microbial impacts, and acting as a signaling interface between the environment and the body. The epidermis, the outermost level of skin, expresses a wide range of proteins to achieve this protective function. Corneocytes in the stratum corneum prevent water loss and external insults (Kolarsick et al., 2011). Although the skin may appear homogeneous, there are subtle anatomic and textural differences from location to location; therefore, the mechanism to maintain a constant epidermal thickness might differ. Also, differences in response to injury and scar formation in different skin sites suggest possible protein variation among them. A recent comparative proteomic analysis comparing human palm with forearm, lower leg, forehead, abdomen, and upper back revealed striking differences between the palm pattern and the others (Rice et al., 2013). Both the palms and soles of the feet have the thickest epidermal layer on the body (Kolarsick et al., 2011), suggesting they might have similar protein profiles. Indeed, we found few differences between proteins of palm and foot. Three structural proteins were distinct in showing higher levels in foot sole than palm. Since the protein pattern from palm exhibits significant differences from other sites, the foot sole evidently does as well.

\subsection{Protein profiles in palm after arsenic exposure}

The lesional palm samples from six arsenic-exposed subjects were analyzed for protein profile in comparison to non-lesion, normal and control palms (Tables S4 and S5). We found two proteins, Fatty Acid Binding Protein 5 (FABP5) and Keratin 6C (KRT6C), were consistently significantly increased in lesion-derived samples compared to other unaffected samples, while the expression level of the other protein, Desmoglein 1 (DSG1), was significantly suppressed (Fig. 3).

Desmoglein 1 (DSG1), a cadherin-like transmembrane glycoprotein, mainly functions by anchoring keratin cytoskeleton to the cell membrane, and plays an important role in promoting keratinocyte differentiation by attenuating MAPK/ERK signaling (Harmon et al., 2013; Samuelov et al., 2013). Down-regulation of DSG1 can lead to loss of cell-cell adhesion, resulting in abnormal epidermal differentiation. Disruption of the DSG1 gene induces various skin diseases (Samuelov et al., 2013). Our result showed decreased DSG1 expression, indicating that the normal epidermal differentiation which requires the choreographed expression of cell adhesion protein may be disturbed after chronic arsenic exposure. Therefore, DSG1 may serve as a potential candidate biomarker and even helpful drug target for skin diseases. Therapeutically increasing the DSG1 expression might promote the recovery of keratinocyte differentiation.

Keratins are intermediate filament proteins responsible for structural reinforcement, and play important roles in maintaining epidermal cell-cell adhesion (Knöbel et al., 2015). Mutation in keratin genes can cause a wide range of cutaneous dermatoses. Mutation in KRT6C is responsible for some cases of pachyonychia congenita (Wilson et al., 2014). In addition to providing structural support in epithelial cells, keratins have also been reported to act as 


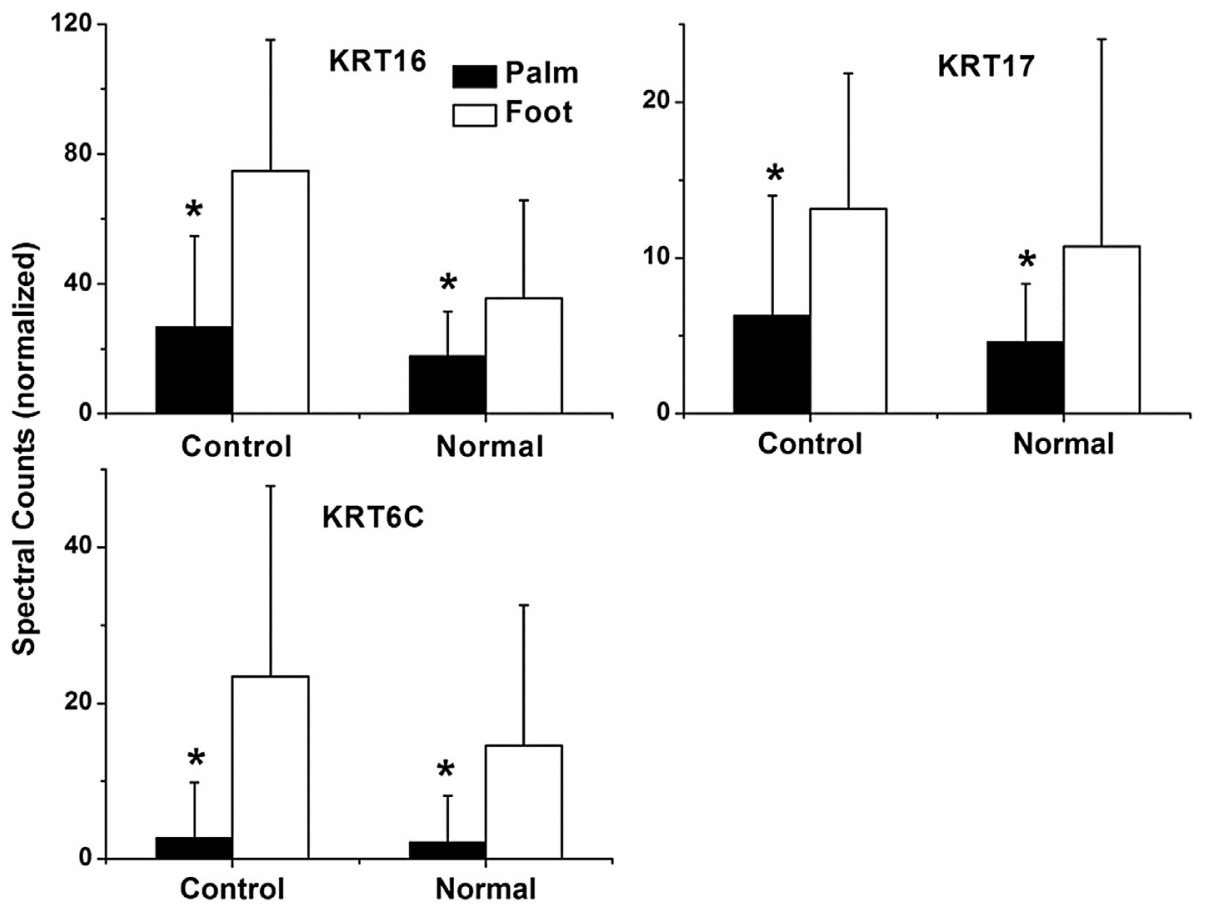

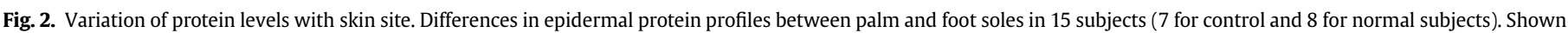
are 3 proteins distinguishing the profile of palm from foot. "** indicates significant differences $(p<0.05)$ between palm and foot.

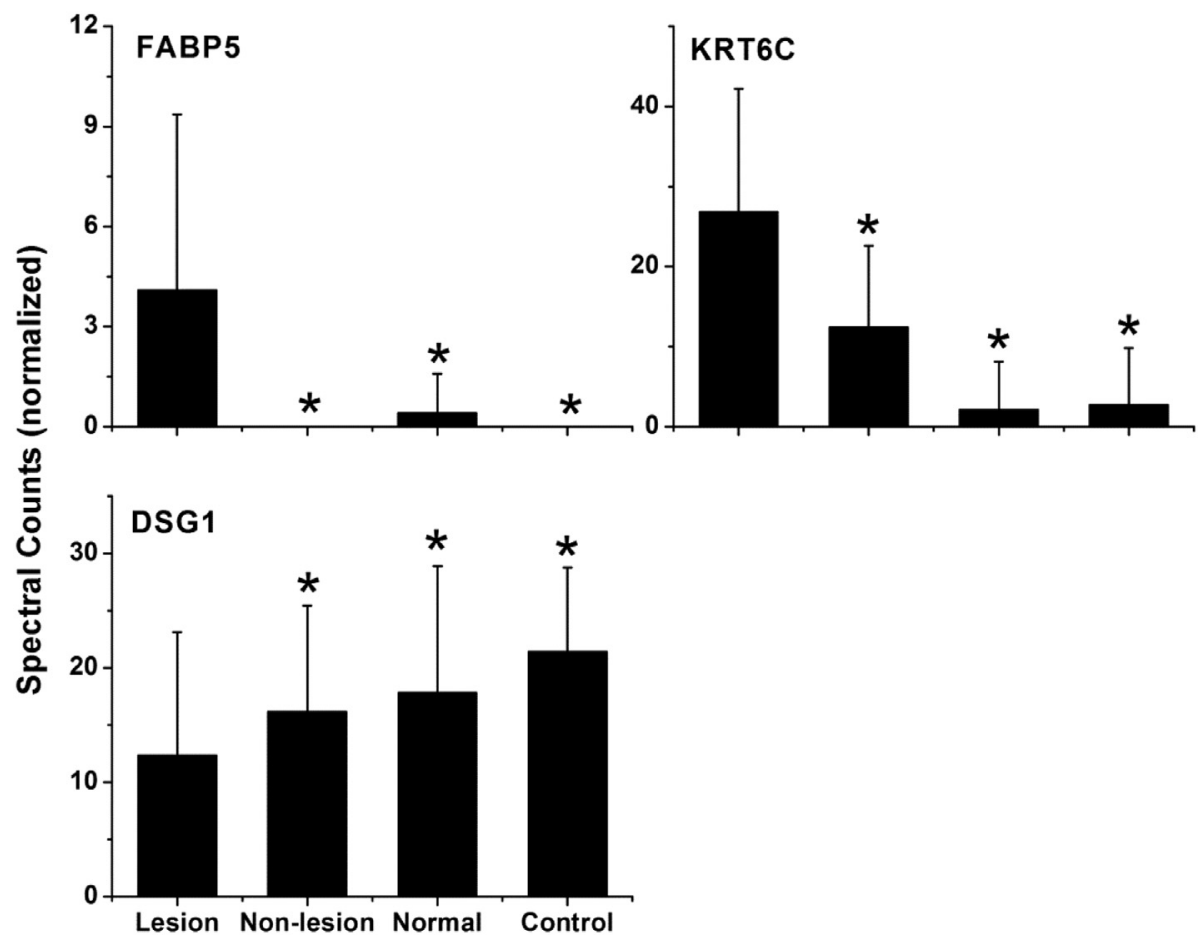

Fig. 3. Different protein levels in lesion, non-lesion, normal and control samples. “*” indicates significant differences ( $p<0.05)$ compared to lesion group.

biomarkers in the diagnosis and prognosis of epithelial-based tumors (Karantza, 2011). Since abnormal expression of KRT6C may affect its structural role in the epidermis, this might represent a biomarker for skin disorder. The molecular pathogenesis of keratins in skin disorders needs to be further elucidated. The fatty acidbinding protein 5 , which was first identified in human epidermal cells, is upregulated in a number of human cancers, including those of prostate (Adamson et al., 2003), bladder (Chen et al., 2011), breast (Levi et al., 2013) and esophagus (Ogawa et al., 2008). Although the mechanisms underlying the upregulation of FABP5 in cancers are incompletely understood, previous studies suggest that FABP5 may promote tumor development. FABP5 plays important 
roles in fatty acid synthesis, transport and metabolism in epidermis which is an active site of fatty acid metabolism (Chmurzyńska, 2006; Furuhashi and Hotamisligil, 2008). Therefore, overexpression of FABP5 may alter the lipid metabolism and thus affect the proliferation or differentiation of keratinocytes. However, further studies are needed to understand the biological roles of these proteins in skin pathogenesis from arsenic exposure.

\section{Conclusion}

Skin diseases can result in specific changes in protein profile in affected areas. Proteomic analysis permits sensitive detection of variation between normal and pathologic tissues, holds excellent potential for biomarker development, and could even identify individuals who are predisposed to certain diseases and aid in therapeutic development (Issaq and Veenstra, 2013). In our studies, three proteins were consistently differentially expressed in lesional skin compared to unaffected skin. A more comprehensive analysis along these lines may reveal the usefulness of these and other proteins as potential molecular markers of chronic arsenic exposure, to detect susceptibility to exposure and to monitor response to arsenic withdrawal or even antidotal therapy.

\section{Acknowledgement}

This work was financially supported by the National Natural Science Foundation of China (grant numbers 21507153, 21277168, 21525730) and the Strategic Priority Research Program of the Chinese Academy of Sciences (grant numbers XDB14030401, XDB14030402). We gratefully acknowledge the financial support provided by the International Postdoctoral Exchange Fellowship Program 2014 by the Office of China Postdoctoral Council (Grant No. 20140052).

\section{Appendix A. Supplementary data}

Supplementary data related to this article can be found at http:// dx.doi.org/10.1016/j.envpol.2016.08.035.

\section{Notes}

The authors declare no competing financial interest.

\section{References}

Abdallah, C., Dumas-Gaudot, E., Renaut, J., Sergeant, K., 2012. Gel-based and gel-free quantitative proteomics approaches at a glance. Int. J. Plant Genom. 2012. Article ID 494572.

Adamson, J., Morgan, E.A., Beesley, C., Mei, Y., Foster, C.S., Fujii, H., Rudland, P.S., Smith, P.H., Ke, Y., 2003. High-level expression of cutaneous fatty acid-binding protein in prostatic carcinomas and its effect on tumorigenicity. Oncogene 22, 2739-2749.

Bolstad, B.M., Irizarry, R.A., Åstrand, M., Speed, T.P., 2003. A comparison of normalization methods for high density oligonucleotide array data based on variance and bias. Bioinform. 19, 185-193.

Chen, R., Feng, C., Xu, Y., 2011. Cyclin-dependent kinase-associated protein Cks2 is associated with bladder cancer progression. J. Int. Med. Res. 39, 533-540.

Chmurzyńska, A., 2006. The multigene family of fatty acid-binding proteins (FABPs): function, structure and polymorphism. J. Appl. Genet. 47, 39-48.

Cui, J.L., Shi, J.B., Jiang, G.B., Jing, C.Y., 2013. Arsenic levels and speciation from ingestion exposures to biomarkers in Shanxi, China: implications for human health. Environ. Sci. Technol. 47, 5419-5424.

Furuhashi, M., Hotamisligil, G.S., 2008. Fatty acid-binding proteins: role in metabolic diseases and potential as drug targets. Nat. Rev. Drug Discov. 7, 489-503.

Guo, H., Wang, Y., Shpeizer, G.M., Yan, S., 2003. Natural occurrence of arsenic in shallow groundwater, Shanyin, Datong Basin, China. J. Environ. Sci. Heal. A 38, 2565-2580.

Harezlak, J., Wu, M.C., Wang, M., Schwartzman, A., Christiani, D.C., Lin, X., 2008 Biomarker discovery for arsenic exposure using functional data. Analysis and feature learning of mass spectrometry proteomic data. J. Proteome Res. 7, $217-224$.

Harmon, R.M., Simpson, C.L., Johnson, J.L., Koetsier, J.L., Dubash, A.D., Najor, N.A. Sarig, O., Sprecher, E., Green, K.J., 2013. Desmoglein-1/Erbin interaction suppresses ERK activation to support epidermal differentiation. J. Clin. Investig. 123, 1556-1570.

Hegedus, C.M., Skibola, C.F., Warner, M., Skibola, D.R., Alexander, D., Lim, S., Dangleben, N.L., Zhang, L., Clark, M., Pfeiffer, R.M., Steinmaus, C., 2008. Decreased urinary beta-defensin-1 expression as a biomarker of response to arsenic. Toxicol. Sci. 106, 74-82.

Hu, Q., Rice, R.H., Qin, Q., Phinney, B.S., Eigenheer, R.A., Bao, W.J., Zhao, B., 2013. Proteomic analysis of human keratinocyte response to $2,3,7,8$ tetrachlorodibenzo-p-dioxin (TCDD) exposure. J. Proteome Res. 12, 5340-5347.

IARC, 2004. Some drinking-water disinfectants and contaminants, including arsenic. IARC Monogr. Eval. Carcinog. Risks Hum. 84, 1-477. PMID:15645577.

Issaq, H.J., Veenstra, T.D., 2013. Biomarker discovery: study design and execution. In: Haleem, J.I., Timothy, D.V. (Eds.), Proteomic and Metabolomic Approaches to Biomarker Discovery. Academic Press, USA, pp. 1-16.

Karantza, V., 2011. Keratins in health and cancer: more than mere epithelial cell markers. Oncogene 30, 127-138.

Knöbel, M., O'Toole, E.A., Smith, F.J., 2015. Keratins and skin disease. Cell Tissue Res. 360, 583-589.

Kolarsick, P.A., Kolarsick, M.A., Goodwin, C., 2011. Anatomy and physiology of the skin. J. Dermatol. Nurses Assoc. 3, 203-213.

Levi, L., Lobo, G., Doud, M.K., von Lintig, J., Seachrist, D., Tochtrop, G.P., Noy, N., 2013. Genetic ablation of the fatty acid-binding protein FABP5 suppresses HER2induced mammary tumorigenesis. Cancer Res. 73, 4770-4780.

Liu, J., Zheng, B., Aposhian, H.V., Zhou, Y., Chen, M.L., Zhang, A., Waalkes, M.P., 2002. Chronic arsenic poisoning from burning high-arsenic-containing coal in Guizhou, China. Environ. Health Perspect. 110, 119-122.

Matschullat, J., 2000. Arsenic in the geosphere-a review. Sci. Total Environ. 249, $297-312$.

Mead, M.N., 2005. Arsenic: in search of an antidote to a global poison. Environ. Health Perspect. 113, A378-A386.

Ogawa, R., Ishiguro, H., Kuwabara, Y., Kimura, M., Mitsui, A., Mori, Y., Mori, R., Tomoda, K., Katada, T., Harada, K., Fujii, Y., 2008. Identification of candidate genes involved in the radiosensitivity of esophageal cancer cells by microarray analysis. Dis. Esophagus 21, 288-297.

Rice, R.H., Bradshaw, K.M., Durbin-Johnson, B.P., Rocke, D.M., Eigenheer, R.A., Phinney, B.S., Schmuth, M., Gruber, R., 2013. Distinguishing ichthyoses by protein profiling. PLoS One 8, e75355.

Samuelov, L., Sarig, O., Harmon, R.M., Rapaport, D., Ishida-Yamamoto, A., Isakov, O., Koetsier, J.L., Gat, A., Goldberg, I., Bergman, R., Spiegel, R., Eytan, O., Geller, S., Peleg, S., Shomron, N., Goh, C.S.M., Wilson, N.J., Smith, F.J.D., Pohler, E., Simpson, M.A., McLean, W.H.I., Irvine, A.D., Horowitz, M., McGrath, J.A., Green, K.J., Sprecher, E., 2013. Desmoglein 1 deficiency results in severe dermatitis, multiple allergies and metabolic wasting. Nat. Genet. 45, $1244-1248$.

Tokar, E.J., Waalkes, M.P., 2011. Arsenic, stem cells, and the developmental basis of adult cancer. Toxicol. Sci. 120, S192-S203.

Vlaanderen, J., Moore, L.E., Smith, M.T., Lan, Q., Zhang, L., Skibola, C.F., Rothman, N., Vermeulen, R., 2010. Application of OMICS technologies in occupational and environmental health research; current status and projections. Occup. Environ. Med. 67, 136-143.

Wang, J.P., Qi, L., Moore, M.R., Ng, J.C., 2002. A review of animal models for the study of arsenic carcinogenesis. Toxicol. Lett. 133, 17-31.

Wilson, N.J., O'Toole, E.A., Milstone, L.M., 2014. The molecular genetic analysis of the expanding pachyonychia congenita case collection. Br. J. Dermatol. 171, 343-355.

Yu, H.S., Chiou, K.S., Chen, G.S., Yang, R.C., Chang, S.F., 1993. Progressive alterations of cytokeratin expressions in the process of chronic arsenism. J. Dermatol. 20, $741-745$.

Yu, H.S., Liao, W.T., Chai, C.Y., 2006. Arsenic carcinogenesis in the skin. J. Biomed. Sci. $13,657-666$

Zhai, R., Su, S., Lu, X., Liao, R., Ge, X., He, M., Huang, Y., Mai, S., Lu, X., Christiani, D. 2005. Proteomic profiling in the sera of workers occupationally exposed to arsenic and lead: identification of potential biomarkers. Biometals 18, 603-613.

Zhang, Y., Fonslow, B.R., Shan, B., Baek, M.C., Yates III, J.R., 2013. Protein analysis by shotgun/bottom-up proteomics. Chem. Rev. 113, 2343-2394. 